\title{
ON THE GROWTH OF SOLUTIONS OF SOME HIGHER ORDER LINEAR DIFFERENTIAL EQUATIONS WITH MEROMORPHIC COEFFICIENTS
}

\author{
M. SAIDANI, B. BELAÏDI
}

\begin{abstract}
In this paper, by using the value distribution theory, we study the growth and the oscillation of meromorphic solutions of the linear differential equation

$$
\begin{aligned}
f^{(k)} & +\left(A_{k-1,1}(z) e^{P_{k-1}(z)}+A_{k-1,2}(z) e^{Q_{k-1}(z)}\right) f^{(k-1)} \\
& +\cdots+\left(A_{0,1}(z) e^{P_{0}(z)}+A_{0,2}(z) e^{Q_{0}(z)}\right) f=F(z),
\end{aligned}
$$

where $A_{j, i}(z)(\not \equiv 0)(j=0, \ldots, k-1), F(z)$ are meromorphic functions of a finite order, and $P_{j}(z), Q_{j}(z)(j=0,1, \ldots, k-1 ; i=1,2)$ are polynomials with degree $n \geqslant 1$. Under some conditions, we prove that as $F \equiv 0$, each meromorphic solution $f \not \equiv 0$ with poles of uniformly bounded multiplicity is of infinite order and satisfies $\rho_{2}(f)=n$ and as $F \not \equiv 0$, there exists at most one exceptional solution $f_{0}$ of a finite order, and all other transcendental meromorphic solutions $f$ with poles of uniformly bounded multiplicities satisfy $\bar{\lambda}(f)=\lambda(f)=\rho(f)=+\infty$ and $\bar{\lambda}_{2}(f)=\lambda_{2}(f)=\rho_{2}(f) \leqslant \max \{n, \rho(F)\}$. Our results extend the previous results due Zhan and Xiao [19].
\end{abstract}

Keywords: Order of growth, hyper-order, exponent of convergence of zero sequence, differential equation, meromorphic function.

Mathematics Subject Classification: 34M10, 30D35

\section{INTRODUCTION AND MAIN RESULTS}

Throughout this paper, we assume that the reader is familiar with the fundamental results and the standard notations of the Nevanlinna's value distribution theory, see [12], [18]. Let $\rho(f)$ stands for the order of growth of a meromorphic function $f$ and the hyper-order of $f$ is defined by

$$
\rho_{2}(f)=\limsup _{r \rightarrow+\infty} \frac{\log \log T(r, f)}{\log r},
$$

where $T(r, f)$ is the Nevanlinna characteristic function of $f$, see [12], [14], [18].

Definition 1.1. (15], [17]) Let $f$ be a meromorphic function. The convergence exponent of the zero-sequence of a meromorphic function $f$ is defined by

$$
\lambda(f)=\limsup _{r \rightarrow+\infty} \frac{\log N\left(r, \frac{1}{f}\right)}{\log r},
$$

M. Saidani and B. Belaïdi, On The Growth of Solutions of Some Higher Order Linear Differential Equations With Meromorphic Coefficients.

(c) Saidani M., Belä̈di B. 2018.

62017. 
where $N\left(r, \frac{1}{f}\right)$ is the integrated counting function of zeros of $f$ in $\{z:|z| \leqslant r\}$, and the exponent of convergence the sequence of distinct zeros of $f$ is defined by

$$
\bar{\lambda}(f)=\limsup _{r \rightarrow+\infty} \frac{\log \bar{N}\left(r, \frac{1}{f}\right)}{\log r}
$$

where $\bar{N}\left(r, \frac{1}{f}\right)$ is the integrated counting function of distinct zeros of $f$ in $\{z:|z| \leqslant r\}$. The hyper convergence exponents of the zero-sequence and the distinct zeros of $f$ are defined respectively by

$$
\lambda_{2}(f)=\limsup _{r \rightarrow+\infty} \frac{\log \log N\left(r, \frac{1}{f}\right)}{\log r}, \quad \bar{\lambda}_{2}(f)=\limsup _{r \rightarrow+\infty} \frac{\log \log \bar{N}\left(r, \frac{1}{f}\right)}{\log r} .
$$

Several authors [3], 99, 14] have study the growth of solutions of the second order linear differential equation

$$
f^{\prime \prime}+A_{1}(z) e^{P(z)} f^{\prime}+A_{2}(z) e^{Q(z)} f=0
$$

where $P(z), Q(z)$ are nonconstant polynomials, $A_{1}(z), A_{2}(z)(\not \equiv 0)$ are entire functions such that $\rho\left(A_{1}\right)<\operatorname{deg} P(z), \rho\left(A_{2}\right)<\operatorname{deg} Q(z)$. Gundersen showed in [9] that if $\operatorname{deg} P(z) \neq \operatorname{deg} Q(z)$, then each nonconstant solution of 1.1$)$ is of infinite order. If $\operatorname{deg} P(z)=\operatorname{deg} Q(z)$, then (1.1) may have nonconstant solutions of a finite order. For instance $f(z)=e^{z}+1$ satisfies $f^{\prime \prime}+e^{z} f^{\prime}-e^{z} f=0$.

In [10], Habib and Belaïdi studied the order and hyper-order of solutions of some higher order linear differential equations and they proved the following result.

Theorem 1.1. (10]) Let $A_{j}(z)(\not \equiv 0), \quad(j=1,2), \quad B_{l}(z)(\not \equiv 0) \quad(l=1, \ldots, k-1), \quad D_{m}$ $(m=0, \ldots, k-1)$ be entire functions with

$$
\max \left\{\rho\left(A_{j}\right), \rho\left(B_{l}\right), \rho\left(D_{m}\right)\right\}<1,
$$

$b_{l}(l=1, \ldots, k-1)$ be complex constants such that $(i) \arg b_{l}=\arg a_{1}$ and $b_{l}=c_{l} a_{1}\left(0<c_{l}<1\right)$ $\left(l \in I_{1}\right)$ and $($ ii $) b_{l}$ is a real constant such that $b_{l} \leqslant 0\left(l \in I_{2}\right)$, where $I_{1} \neq \varnothing, I_{2} \neq \varnothing, I_{1} \cap I_{2}=\varnothing$, $I_{1} \cup I_{2}=\{1,2, \ldots, k-1\}$, and $a_{1}$, $a_{2}$ are complex numbers such that $a_{1} a_{2} \neq 0, a_{1} \neq a_{2}$ (suppose that $\left|a_{1}\right| \leqslant\left|a_{2}\right|$ ). If $\arg a_{1} \neq \pi$ or $a_{1}$ is a real number such that $a_{1}<\frac{b}{1-c}$, where $c=\max \left\{c_{l}: l \in I_{1}\right\}$ and $b=\min \left\{b_{l}: l \in I_{2}\right\}$, then each solution $f \not \equiv 0$ of the equation

$$
\begin{aligned}
f^{(k)} & +\left(D_{k-1}+B_{k-1} e^{b_{k-1} z}\right) f^{(k-1)}+\ldots+\left(D_{1}+B_{1} e^{b_{1} z}\right) f^{\prime} \\
& +\left(D_{0}+A_{1} e^{a_{1} z}+A_{2} e^{a_{2} z}\right) f=0
\end{aligned}
$$

satisfies $\rho(f)=+\infty$ and $\rho_{2}(f)=1$.

And in [2], they studied the order and hyper-order of solutions of some higher order linear differential equations with meromorphic coefficient and they proved the following result.

Theorem 1.2. ([2]) Let $A_{j}(z)(\not \equiv 0)(j=1,2), B_{l}(z)(\not \equiv 0) \quad(l=1, \ldots, k-1)$ be meromorphic functions with

$$
\max \left\{\rho\left(A_{j}\right) \quad(j=1,2), \rho\left(B_{l}\right) \quad(l=1, \ldots, k-1)\right\}<1,
$$

$b_{l} \quad(l=1, \ldots, k-1)$ be complex constants such that $(i) b_{l}=c_{l} a_{1}\left(0<c_{l}<1\right)\left(l \in I_{1}\right)$ and (ii) $b_{l}$ is a real constant such that $b_{l}<0\left(l \in I_{2}\right)$, where $I_{1} \neq \emptyset, I_{2} \neq \emptyset, I_{1} \cap I_{2}=\emptyset$, $I_{1} \cup I_{2}=\{1,2, \ldots, k-1\}$, and $a_{1}, a_{2}$ are complex numbers such that $a_{1} a_{2} \neq 0, a_{1} \neq a_{2}$ (suppose that $\left|a_{1}\right| \leqslant\left|a_{2}\right|$ ). If $\arg a_{1} \neq \pi$ or $a_{1}$ is a real number such that $a_{1}<\frac{b}{1-c}$, where 
$c=\max \left\{c_{l}, l \in I_{1}\right\}$ and $b=\min \left\{b_{l}, l \in I_{2}\right\}$, then each meromorphic solution $f(\not \equiv 0)$ with poles of uniformly bounded multiplicities of the equation

$$
f^{(k)}+B_{k-1} e^{b_{k-1} z} f^{(k-1)}+\cdots+B_{1} e^{b_{1} z} f^{\prime}+\left(A_{1} e^{a_{1} z}+A_{2} e^{a_{2} z}\right) f=0
$$

satisfies $\rho(f)=+\infty$ and $\rho_{2}(f)=1$.

In [19], Zhan and Xiao studied the homogeneous and nonhomogeneous higher order differential equations and obtained the following results.

Theorem 1.3. ([19]) Let $A_{j i}(z)(\not \equiv 0)$ be entire functions with $\rho\left(A_{j i}\right)<n, n \geqslant 1$ is a positive integer, $j=0,1, \ldots, k-1 ; i=1,2$. Let $P_{j}(z)=a_{j, n} z^{n}+\cdots+a_{j, 0}$ and $Q_{j}(z)=b_{j, n} z^{n}+\cdots+b_{j, 0}$ be polynomials, where $a_{j, q}, b_{j, q}(j=0,1, \ldots, k-1 ; q=0,1, \ldots, n)$ are complex numbers such that $a_{j, n} b_{j, n} \neq 0, a_{0, n} \neq b_{0, n}$ and $a_{j, n}=c_{j} a_{0, n}, b_{j, n}=c_{j} b_{0, n}, c_{j}>1$, $j=1, \ldots, k-1$ are distinct numbers. Then each solution $f(\not \equiv 0)$ of the equation

$$
\begin{aligned}
f^{(k)} & +\left(A_{k-1,1}(z) e^{P_{k-1}(z)}+A_{k-1,2}(z) e^{Q_{k-1}(z)}\right) f^{(k-1)} \\
& +\cdots+\left(A_{0,1}(z) e^{P_{0}(z)}+A_{0,2}(z) e^{Q_{0}(z)}\right) f=0
\end{aligned}
$$

of a finite order.

Theorem 1.4. ([19]) Let $A_{j i}(z)$ ( $\left.\not \equiv 0\right)$ be entire functions with $\rho\left(A_{j i}\right)<n$, where $n \geqslant 1$ is a positive integer, $j=0,1, \ldots, k-1 ; i=1,2$. Let $P_{j}(z)=a_{j, n} z^{n}+\cdots+a_{j, 0}$ and $Q_{j}(z)=b_{j, n} z^{n}+\cdots+b_{j, 0}$ be polynomials, where $a_{j, q}, b_{j, q}(j=0,1, \ldots, k-1 ; q=0,1, \ldots, n)$ are complex numbers such that $a_{j, n} b_{j, n} \neq 0, a_{0, n} \neq b_{0, n}$ and $a_{j, n}=c_{j} a_{0, n}, b_{j, n}=c_{j} b_{0, n}, c_{j}>1$, $j=1, \ldots, k-1$ are distinct numbers. $F(z)(\not \equiv 0)$ is an entire function of a finite order. Then the equation

$$
\begin{aligned}
f^{(k)} & +\left(A_{k-1,1}(z) e^{P_{k-1}(z)}+A_{k-1,2}(z) e^{Q_{k-1}(z)}\right) f^{(k-1)} \\
& +\cdots+\left(A_{0,1}(z) e^{P_{0}(z)}+A_{0,2}(z) e^{Q_{0}(z)}\right) f=F(z)
\end{aligned}
$$

satisfies the following statements:

(i) There exists at most one exceptional solution $f_{0}$ of a finite order, and all other solutions satisfy $\bar{\lambda}(f)=\lambda(f)=\rho(f)=+\infty$ and $\bar{\lambda}_{2}(f)=\lambda_{2}(f)=\rho_{2}(f) \leqslant \max \{n, \rho(F)\}$.

(ii) If there exists $f_{0}$ of a finite order, then $\rho\left(f_{0}\right) \leqslant \max \left\{n, \bar{\lambda}\left(f_{0}\right), \rho(F)\right\}$.

(iii) If $F(z)$ is an entire function of order less than $n$ and $\arg a_{0, n} \neq \arg b_{0, n}$, then each solution of (1.5) is of infinite order.

In this paper, we are concerned with a more general problem. We extend and improve Theorem 1.3 and Theorem 1.4. In fact, we will prove the following theorems.

Theorem 1.5. Let $A_{j i}(z)(\not \equiv 0)$ be meromorphic functions of a finite order such that $\max \left\{\rho\left(A_{j i}\right), j=0,1, \ldots, k-1 ; i=1,2\right\}<n$, where $n \geqslant 1$ is a positive integer. Let $P_{j}(z)=a_{j, n} z^{n}+\cdots+a_{j, 0}$ and $Q_{j}(z)=b_{j, n} z^{n}+\cdots+b_{j, 0}$ be polynomials, where $a_{j, q}, b_{j, q}$ $(j=0,1, \ldots, k-1 ; q=0,1, \ldots, n)$ are complex numbers such that $a_{j, n} b_{j, n} \neq 0, a_{0, n} \neq b_{0, n}$ and $a_{j, n}=c_{j} a_{0, n}, b_{j, n}=c_{j} b_{0, n}, c_{j}>1, j=1, \ldots, k-1$ are distinct numbers. Then each meromorphic solution $f(\not \equiv 0)$ of equation (1.4) with poles of uniformly bounded multiplicity is of infinite order and satisfies $\rho_{2}(f)=n$.

Theorem 1.6. Let $A_{j i}(z)(\not \equiv 0), F(z)(\not \equiv 0)$ be meromorphic functions of a finite order with $\max \left\{\rho\left(A_{j i}\right), j=0,1, \ldots, k-1 ; i=1,2\right\}<n$, where $n \geqslant 1$ is a positive integer. Let $P_{j}(z)=a_{j, n} z^{n}+\cdots+a_{j, 0}$ and $Q_{j}(z)=b_{j, n} z^{n}+\cdots+b_{j, 0}$ be polynomials, where $a_{j, q}, b_{j, q}$ $(j=0,1, \ldots, k-1 ; q=0,1, \ldots, n)$ are complex numbers such that $a_{j, n} b_{j, n} \neq 0, a_{0, n} \neq b_{0, n}$ and $a_{j, n}=c_{j} a_{0, n}, b_{j, n}=c_{j} b_{0, n}, c_{j}>1, j=1, \ldots, k-1$ are distinct numbers. Then the equation (1.5) satisfies: 
(i) There exists at most one exceptional meromorphic solution $f_{0}$ with finite order, and all other transcendental meromorphic solutions $f$ with poles of uniformly bounded multiplicities satisfy

$$
\bar{\lambda}(f)=\lambda(f)=\rho(f)=+\infty
$$

and

$$
\bar{\lambda}_{2}(f)=\lambda_{2}(f)=\rho_{2}(f) \leqslant \max \{n, \rho(F)\} .
$$

(ii) If there exists $f_{0}$ of a finite order, then $\rho\left(f_{0}\right) \leqslant \max \left\{n, \bar{\lambda}\left(f_{0}\right), \rho(F)\right\}$.

(iii) If $F(z)$ is a meromorphic function of order less than $n$ and $\arg a_{0, n} \neq \arg b_{0, n}$, then each meromorphic solution $f$ of (1.5) with poles of uniformly bounded multiplicities is of infinite order and satisfies $\rho_{2}(f)=n$.

\section{Auxiliary Lemmata}

First, we recall the following definitions. The linear measure of a set $E \subset[0,+\infty)$ is defined as

$$
m(E)=\int_{0}^{+\infty} \chi_{E}(t) d t
$$

and the logarithmic measure of a set $F \subset[1,+\infty)$ is defined by

$$
\operatorname{lm}(F)=\int_{1}^{+\infty} \frac{\chi_{F}(t)}{t} d t
$$

where $\chi_{H}(t)$ is the characteristic function of a set $H$.

Lemma 2.1. (1] $)$ Let $P_{j}(z)(j=0,1, \ldots, k)$ be polynomials with $\operatorname{deg} P_{0}=n(n \geqslant 1)$ and $\operatorname{deg} P_{j} \leqslant n(j=1, \ldots, k)$. Let $A_{j}(z)(j=0,1, \ldots, k)$ be meromorphic functions of a finite order and $\max \left\{\rho\left(A_{j}\right), j=0,1, \ldots, k\right\}<n$ such that $A_{0}(z) \not \equiv 0$. We denote

$$
F(z)=A_{k} e^{P_{k}(z)}+A_{k-1} e^{P_{k-1}(z)}+\cdots+A_{1} e^{P_{1}(z)}+A_{0} e^{P_{0}(z)} .
$$

If $\operatorname{deg}\left(P_{0}(z)-P_{j}(z)\right)=n$ for all $j=1, \ldots, k$, then $F$ is a nontrivial meromophic function with finite order satisfying $\rho(F)=n$.

Lemma 2.2. ([8]) Let $f(z)$ be a transcendental meromorphic function and let $\alpha>1$ and $\varepsilon>0$ be given constants. Then there exist a set $E_{1} \subset(1,+\infty)$ of a finite logarithmic measure and a constant $B>0$ that depends only on $\alpha$ and positive integers $(n, m)$ obeying $n>m \geqslant 0$ such that for all $z$ satisfying $|z|=r \notin[0,1] \cup E_{1}$, we have

$$
\left|\frac{f^{(n)}(z)}{f^{(m)}(z)}\right| \leqslant B\left[\frac{T(\alpha r, f)}{r}\left(\log ^{\alpha} r\right) \log T(\alpha r, f)\right]^{n-m} .
$$

Lemma 2.3. (11]) Let $P(z)=(\alpha+i \beta) z^{n}+\cdots(\alpha$, $\beta$ are real numbers, $|\alpha|+|\beta| \neq 0)$ be a polynomial with degree $n \geqslant 1$ and $A(z)$ be a meromorphic function with $\rho(A)<n$. Let

$$
f(z)=A(z) e^{P(z)}, \quad z=r e^{i \theta}, \quad \delta(P, \theta)=\alpha \cos n \theta-\beta \sin n \theta .
$$

Then for any given $\varepsilon>0$, there exists a set $E_{2} \subset[1,+\infty)$ of a finite logarithmic measure such that for each $\theta \in[0,2 \pi) \backslash H(H=\{\theta \in[0,2 \pi): \delta(P, \theta)=0\})$ and for $|z|=r \notin[0,1] \cup E_{2}$, $r \rightarrow+\infty$, we have

(i) if $\delta(P, \theta)>0$, then

$$
\exp \left\{(1-\varepsilon) \delta(P, \theta) r^{n}\right\} \leqslant\left|f\left(r e^{i \theta}\right)\right| \leqslant \exp \left\{(1+\varepsilon) \delta(P, \theta) r^{n}\right\},
$$

(ii) if $\delta(P, \theta)<0$, then

$$
\exp \left\{(1+\varepsilon) \delta(P, \theta) r^{n}\right\} \leqslant\left|f\left(r e^{i \theta}\right)\right| \leqslant \exp \left\{(1-\varepsilon) \delta(P, \theta) r^{n}\right\} .
$$


Lemma 2.4. ([5]) Let $f(z)$ be a meromorphic function of order $\rho(f)=\rho<+\infty$. Then for any given $\varepsilon>0$, there exists a set $E_{3} \subset(1,+\infty)$ that has finite linear measure and finite logarithmic measure such that as $|z|=r \notin[0,1] \cup E_{3}, r \rightarrow+\infty$, we have $|f(z)| \leqslant \exp \left(r^{\rho+\varepsilon}\right)$.

It is well known that due to the Wiman-Valiron theory [13], [15], it is important to studyt the properties of entire solutions of differential equations. In [4], Chen extended the WimanValiron theory from entire functions to meromorphic functions. Here we give a special form of the result given by Wang and Yi in [17], when meromorphic function has infinite order.

Let $g(z)=\sum_{n=0}^{\infty} a_{n} z^{n}$ be an entire function. By $\mu(r)=\max \left\{\left|a_{n}\right| r^{n} ; n=0,1, \ldots\right\}$ we denote the maximum term of $g$ and by $\nu_{g}(r)=\max \left\{m: \mu(r)=\left|a_{m}\right| r^{m}\right\}$ we denote the central index of $g$.

Lemma 2.5. ([17]) Let $f(z)=g(z) / d(z)$ be a meromorphic function of infinite order obeying $\rho_{2}(f)=\sigma, g(z)$ and $d(z)$ are entire functions, where $\rho(d)<+\infty$. Then there exists a sequence of complex numbers $\left\{z_{m}=r_{m} e^{i \theta_{m}}\right\}_{m \in \mathbb{N}}$ satisfying

$$
r_{m} \rightarrow+\infty, \quad \theta_{m} \in[0,2 \pi) ; \quad m \in \mathbb{N}, \quad \lim _{m \rightarrow+\infty} \theta_{m}=\theta_{0} \in[0,2 \pi), \quad\left|g\left(z_{m}\right)\right|=M\left(r_{m}, g\right)
$$

and for sufficiently large $m$ we have

$$
\begin{aligned}
& \frac{f^{(n)}\left(z_{m}\right)}{f\left(z_{m}\right)}=\left(\frac{\nu_{g}\left(r_{m}\right)}{z_{m}}\right)^{n}(1+o(1)) \quad(n \in \mathbb{N}), \\
& \limsup _{r_{m} \rightarrow+\infty} \frac{\log \log \nu_{g}\left(r_{m}\right)}{\log r_{m}}=\rho_{2}(g)=\sigma .
\end{aligned}
$$

Lemma 2.6. ([9]) Let $\varphi:[0,+\infty) \rightarrow \mathbb{R}$ and $\psi:[0,+\infty) \rightarrow \mathbb{R}$ be a monotone nondecreasing functions such that $\varphi(r) \leqslant \psi(r)$ for all $r \notin\left(E_{4} \cup[0,1]\right)$, where $E_{4}$ is a set of a finite logarithmic measure. Let $\alpha>1$ be a given constant. Then there exists an $r_{1}=r_{1}(\alpha)>0$ such that $\varphi(r) \leqslant \psi(\alpha r)$ for all $r>r_{1}$.

Lemma 2.7. Suppose that $k \geqslant 2$ and $F, A_{0}, A_{1}, \ldots, A_{k-1}$ are meromorphic functions such that $\rho=\max \left\{\rho\left(A_{j}\right) j=0,1,2, \ldots, k-1, \rho(F)\right\}<+\infty$. Let $f(z)$ be a transcendental meromorphic solution with all poles of $f$ are of uniformly bounded multiplicity, of equation

$$
f^{(k)}+A_{k-1} f^{(k-1)}+\cdots+A_{1} f^{\prime}+A_{0} f=F .
$$

Then $\rho_{2}(f) \leqslant \rho$.

Proof. We assume that $f$ is a transcendental meromorphic solution of equation (2.1). If $\rho(f)<+\infty$, then $\rho_{2}(f)=0 \leqslant \rho$. Assume that $f$ is a meromorphic solution to equation (2.1) of infinite order with poles of uniformly bounded multiplicity. By (2.1) we have

$$
\left|\frac{f^{(k)}}{f}\right| \leqslant\left|A_{k-1}(z)\right|\left|\frac{f^{(k-1)}}{f}\right|+\cdots+\left|A_{1}(z)\right|\left|\frac{f^{\prime}}{f}\right|+\left|\frac{F}{f}\right|+\left|A_{0}(z)\right| .
$$

By (2.1) it follows that the poles of $f$ can locate only at the poles of $A_{j}(j=0, \ldots, k-1)$ and $F$. Note that the poles of $f$ are of uniformly bounded multiplicity. Hence, $\lambda(1 / f) \leqslant \rho$. By the Hadamard factorization theorem, we know that $f$ can be expressed as $f(z)=\frac{g(z)}{d(z)}$, where $g(z)$ and $d(z)$ are entire functions with

$$
\lambda(d)=\rho(d)=\lambda(1 / f) \leqslant \rho<\rho(f)=\rho(g)=+\infty
$$

and $\rho_{2}(f)=\rho_{2}(g)$. By Lemma 2.5, there exists a sequence $\left\{z_{m}=r_{m} e^{i \theta_{m}}\right\}_{m \in \mathbb{N}}$ satisfying

$$
r_{m} \rightarrow+\infty, \quad \theta_{m} \in[0,2 \pi), \quad \lim _{m \rightarrow+\infty} \theta_{m}=\theta_{0} \in[0,2 \pi), \quad\left|g\left(z_{m}\right)\right|=M\left(r_{m}, g\right)
$$


such that for $m$ sufficiently large we have

$$
\frac{f^{(n)}\left(z_{m}\right)}{f\left(z_{m}\right)}=\left(\frac{\nu_{g}\left(r_{m}\right)}{z_{m}}\right)^{n}(1+o(1))(n \in \mathbb{N})
$$

and

$$
\limsup _{r_{m} \rightarrow+\infty} \frac{\log \log \nu_{g}\left(r_{m}\right)}{\log r_{m}}=\rho_{2}(g)
$$

By Lemma 2.4. for each given $\varepsilon>0$, there exists a set $E_{3} \subset(1,+\infty)$ of a finite logarithmic measure such that

$$
|F(z)| \leqslant \exp \left\{r^{\rho+\varepsilon}\right\},|d(z)| \leqslant \exp \left\{r^{\rho+\varepsilon}\right\}
$$

and

$$
\left|A_{j}(z)\right| \leqslant \exp \left\{r^{\rho+\varepsilon}\right\} \quad(j=0, \ldots, k-1)
$$

hold for $|z|=r \notin[0,1] \cup E_{3}, r \rightarrow+\infty$. Since $M(r, g) \geqslant 1$ for $r$ sufficiently large, it follows from (2.5) that

$$
\left|\frac{F(z)}{f(z)}\right|=\frac{|F(z)||d(z)|}{|g(z)|}=\frac{|F(z)||d(z)|}{M(r, g)} \leqslant \exp \left\{2 r^{\rho+\varepsilon}\right\} .
$$

Substituting (2.3), 2.6) and (2.7) into (2.2), we obtain

$$
\left(\frac{\nu_{g}\left(r_{m}\right)}{r_{m}}\right)^{k}|1+o(1)| \leqslant \sum_{j=1}^{k-1} e^{r_{m}^{\rho+\varepsilon}}\left(\frac{\nu_{g}\left(r_{m}\right)}{r_{m}}\right)^{j}|1+o(1)|+e^{r_{m}^{\rho+\varepsilon}}+e^{2 r_{m}^{\rho+\varepsilon}}
$$

It follows that

$$
\left(\nu_{g}\left(r_{m}\right)\right)^{k}|1+o(1)| \leqslant(k+1) e^{2 r_{m}^{\rho+\varepsilon}} r_{m}^{k}\left(\nu_{g}\left(r_{m}\right)\right)^{k-1}|1+o(1)|
$$

Hence,

$$
\nu_{g}\left(r_{m}\right) \leqslant(k+1) A r_{m}^{k} e^{2 r_{m}^{\rho+\varepsilon}}
$$

where the sequence $\left\{z_{m}=r_{m} e^{i \theta_{m}}\right\}_{m \in \mathbb{N}}$ satisfies

$r_{m} \notin[0,1] \cup E_{3}, \quad r_{m} \rightarrow+\infty, \quad \theta_{m} \in[0,2 \pi), \quad \lim _{m \rightarrow+\infty} \theta_{m}=\theta_{0} \in[0,2 \pi), \quad\left|g\left(z_{m}\right)\right|=M\left(r_{m}, g\right)$

and $A>0$ is some constant. Then by (2.8), Lemma 2.6 and $\varepsilon>0$ being arbitrary, we obtain that $\rho_{2}(g)=\rho_{2}(f) \leqslant \rho$.

Remark 2.1. For $F \equiv 0$, Lemma 2.7 was proved by Chen and $\mathrm{Xu}$ in [7].

Lemma 2.8. ([16]) Let $g(z)$ be a transcendental entire function and $\nu_{g}(r)$ be the central index of $g$. For each sufficiently large $|z|=r$, let $z_{r}=r e^{i \theta_{r}}$ be a point satisfying $\left|g\left(z_{r}\right)\right|=M(r, g)$. Then there exist a constant $\delta_{r}(>0)$ and a set $E_{5}$ of a finite logarithmic measure such that for all $z$ satisfying $|z|=r \notin E_{5}$ and $\arg z=\theta \in\left[\theta_{r}-\delta_{r}, \theta_{r}+\delta_{r}\right]$, we have

$$
\frac{g^{(n)}(z)}{g(z)}=\left(\frac{\nu_{g}(r)}{z}\right)^{n}(1+o(1)) \quad(n \geqslant 1 \text { is an integer }) \text {. }
$$

Lemma 2.9. (8]) Let $f(z)$ be a transcendental meromorphic function of a finite order $\rho$. Let $\Gamma=\left\{\left(k_{1}, j_{1}\right),\left(k_{2}, j_{2}\right), \ldots,\left(k_{m}, j_{m}\right)\right\}$ denote a set of distinct pairs of integers satisfying $k_{i}>j_{i} \geqslant 0(i=1,2, \ldots, m)$ and let $\varepsilon>0$ be a given constant. Then there exists a set $E_{6} \subset[1,+\infty)$ of a finite logarithmic measure such that for all z obeying $|z|=r \notin[0,1] \cup E_{6}$ and $(k, j) \in \Gamma$, we have

$$
\left|\frac{f^{(k)}(z)}{f^{(j)}(z)}\right| \leqslant|z|^{(k-j)(\rho-1+\varepsilon)}
$$


Lemma 2.10. Let $f(z)=g(z) / d(z)$ be a meromorphic function with $\rho(f)=\rho \leqslant+\infty$, where $g(z)$ and $d(z)$ are entire functions satisfying one of the following conditions:

(i) $g$ is transcendental and $d$ is polynomial,

(ii) $g$, $d$ are transcendental and $\lambda(d)=\rho(d)=\beta<\rho(g)=\rho$.

For each sufficiently large $|z|=r$, let $z_{r}=r e^{i \theta_{r}}$ be a point satisfying $\left|g\left(z_{r}\right)\right|=M(r, g)$ and let $\nu_{g}(r)$ be the central index of $g$. Then there exist a constant $\delta_{r}(>0)$, a sequence $\left\{r_{m}\right\}_{m \in \mathbb{N}}$, $r_{m} \rightarrow+\infty$ and a set $E_{7}$ of finite logarithmic measure such that the estimation

$$
\frac{f^{(n)}(z)}{f(z)}=\left(\frac{\nu_{g}\left(r_{m}\right)}{z}\right)^{n}(1+o(1)) \quad(n \geqslant 1 \text { is an integer })
$$

holds for all z satisfying $|z|=r_{m} \notin E_{7}, r_{m} \rightarrow+\infty$ and $\arg z=\theta \in\left[\theta_{r}-\delta_{r}, \theta_{r}+\delta_{r}\right]$.

Proof. By mathematical induction, we obtain

$$
f^{(n)}=\frac{g^{(n)}}{d}+\sum_{j=0}^{n-1} \frac{g^{(j)}}{d} \sum_{\left(j_{1} \cdots j_{n}\right)} C_{j j_{1} \cdots j_{n}}\left(\frac{d^{\prime}}{d}\right)^{j_{1}} \cdots\left(\frac{d^{(n)}}{d}\right)^{j_{n}},
$$

where $C_{j j_{1} \cdots j_{n}}$ are constants and $j+j_{1}+2 j_{2}+\cdots+n j_{n}=n$. Hence,

$$
\frac{f^{(n)}}{f}=\frac{g^{(n)}}{g}+\sum_{j=0}^{n-1} \frac{g^{(j)}}{g} \sum_{\left(j_{1} \cdots j_{n}\right)} C_{j j_{1} \cdots j_{n}}\left(\frac{d^{\prime}}{d}\right)^{j_{1}} \cdots\left(\frac{d^{(n)}}{d}\right)^{j_{n}} .
$$

For each sufficiently large $|z|=r$, let $z_{r}=r e^{i \theta_{r}}$ be a point satisfying $\left|g\left(z_{r}\right)\right|=M(r, g)$. By Lemma 2.8, there exist a constant $\delta_{r}(>0)$ and a set $E_{5}$ of a finite logarithmic measure such that for all $z$ obeying $|z|=r \notin E_{5}$ and $\arg z=\theta \in\left[\theta_{r}-\delta_{r}, \theta_{r}+\delta_{r}\right]$, we have

$$
\frac{g^{(j)}(z)}{g(z)}=\left(\frac{\nu_{g}(r)}{z}\right)^{j}(1+o(1))(j=1,2, \ldots, n),
$$

where $\nu_{g}(r)$ is the central index of $g$. Substituting (2.11) into 2.10 yields

$$
\begin{aligned}
\frac{f^{(n)}(z)}{f(z)}= & \left(\frac{\nu_{g}(r)}{z}\right)^{n}[(1+o(1)) \\
& \left.+\sum_{j=0}^{n-1}\left(\frac{\nu_{g}(r)}{z}\right)^{j-n}(1+o(1)) \sum_{\left(j_{1} \cdots j_{n}\right)} C_{j j_{1} \cdots j_{n}}\left(\frac{d^{\prime}}{d}\right)^{j_{1}} \cdots\left(\frac{d^{(n)}}{d}\right)^{j_{n}}\right] .
\end{aligned}
$$

We can choose a constant $\sigma$ such that $\beta<\sigma<\rho$. By Lemma 2.9, for any given $\varepsilon$ $(0<2 \varepsilon<\sigma-\beta)$, we have

$$
\left|\frac{d^{(s)}(z)}{d(z)}\right| \leqslant r^{s(\beta-1+\varepsilon)}(s=1,2, \ldots, n),
$$

where $|z|=r \notin[0,1] \cup E_{6}, E_{6} \subset(1,+\infty)$ with $\operatorname{lm}\left(E_{6}\right)<+\infty$. From this and $j_{1}+2 j_{2}+\cdots+n j_{n}=n-j$, we have

$$
|z|^{n-j}\left|\left(\frac{d^{\prime}}{d}\right)^{j_{1}} \cdots\left(\frac{d^{(n)}}{d}\right)^{j_{n}}\right| \leqslant|z|^{(n-j)(\beta+\varepsilon)}
$$

for $|z|=r \notin[0,1] \cup E_{6}$. By $\rho(g)=\rho$, there exists a sequence $\left\{r_{m}^{\prime}\right\}\left(r_{m}^{\prime} \rightarrow+\infty\right)$ satisfying

$$
\lim _{r_{m}^{\prime} \rightarrow+\infty} \frac{\log \nu_{g}\left(r_{m}^{\prime}\right)}{\log r_{m}^{\prime}}=\rho .
$$


Setting the logarithmic measure of $E_{7}=[0,1] \cup E_{5} \cup E_{6}, \operatorname{lm}\left(E_{7}\right)=\delta<+\infty$, there exists a point $r_{m} \in\left[r_{m}^{\prime},(\delta+1) r_{m}^{\prime}\right] \backslash E_{7}$. Since

$$
\frac{\log \nu_{g}\left(r_{m}\right)}{\log r_{m}} \geqslant \frac{\log \nu_{g}\left(r_{m}^{\prime}\right)}{\log \left[(\delta+1) r_{m}^{\prime}\right]}=\frac{\log \nu_{g}\left(r_{m}^{\prime}\right)}{\left(\log r_{m}^{\prime}\right)\left[1+\frac{\log (\delta+1)}{\log r_{m}^{\prime}}\right]}
$$

we get

$$
\lim _{r_{m} \rightarrow+\infty} \frac{\log \nu_{g}\left(r_{m}\right)}{\log r_{m}}=\rho .
$$

Hence, for sufficiently large $m$, we obtain

$$
\nu_{g}\left(r_{m}\right) \geqslant r_{m}^{\rho-\varepsilon} \geqslant r_{m}^{\sigma-\varepsilon},
$$

where $\rho-\varepsilon$ can be replaced by a large enough number $M$ if $\rho=+\infty$. This and (2.14) imply

$$
\left|\left(\frac{\nu_{g}(r)}{z}\right)^{j-n}\left(\frac{d^{\prime}}{d}\right)^{j_{1}} \cdots\left(\frac{d^{(n)}}{d}\right)^{j_{n}}\right| \leqslant r_{m}^{(n-j)(\beta-\sigma+2 \varepsilon)} \rightarrow 0, r_{m} \rightarrow+\infty,
$$

where $|z|=r_{m} \notin E_{7}$ and $\arg z=\theta \in\left[\theta_{r}-\delta_{r}, \theta_{r}+\delta_{r}\right]$. From (2.12) and (2.19), we obtain our result.

Lemma 2.11. Let $f(z)=g(z) / d(z)$ be a meromorphic function with $\rho(f)=\rho \leqslant+\infty$, where $g(z)$ and $d(z)$ are entire functions satisfying one of the following conditions

(i) $g$ is transcendental and $d$ is polynomial,

(ii) $g$, $d$ are transcendental and $\lambda(d)=\rho(d)=\beta<\rho(g)=\rho$.

For each sufficiently large $|z|=r$, let $z_{r}=r e^{i \theta_{r}}$ be a point satisfying $\left|g\left(z_{r}\right)\right|=M(r, g)$. Then there exist a constant $\delta_{r}(>0)$, a sequence $\left\{r_{m}\right\}_{m \in \mathbb{N}}, r_{m} \rightarrow+\infty$ and a set $E_{8}$ of a finite logarithmic measure such that the estimate

$$
\left|\frac{f(z)}{f^{(n)}(z)}\right| \leqslant r_{m}^{2 n} \quad(n \geqslant 1 \text { is an integer })
$$

holds for all z satisfying $|z|=r_{m} \notin E_{8}, r_{m} \rightarrow+\infty$ and $\arg z=\theta \in\left[\theta_{r}-\delta_{r}, \theta_{r}+\delta_{r}\right]$.

Proof. Let $z_{r}=r e^{i \theta_{r}}$ be a point satisfying $\left|g\left(z_{r}\right)\right|=M(r, g)$. By Lemma 2.10, there exist a constant $\delta_{r}(>0)$, a sequence $\left\{r_{m}\right\}_{m \in \mathbb{N}}, r_{m} \rightarrow+\infty$ and a set $E_{8}$ of a finite logarithmic measure such that the estimate

$$
\frac{f^{(n)}(z)}{f(z)}=\left(\frac{\nu_{g}\left(r_{m}\right)}{z}\right)^{n}(1+o(1)) \quad(n \geqslant 1 \text { is an integer })
$$

holds for all $z$ satisfying $|z|=r_{m} \notin E_{8}, r_{m} \rightarrow+\infty$ and $\arg z=\theta \in\left[\theta_{r}-\delta_{r}, \theta_{r}+\delta_{r}\right]$. On the other hand, for any given $\varepsilon>0$ and sufficiently large $m$ we obtain

$$
\nu_{g}\left(r_{m}\right) \geqslant r_{m}^{\rho-\varepsilon}
$$

where $\rho-\varepsilon$ can be replaced by a large enough number $M$ if $\rho=+\infty$. Hence, we have

$$
\left|\frac{f(z)}{f^{(n)}(z)}\right| \leqslant r_{m}^{2 n}
$$

This completes the proof.

Lemma 2.12. ([12]) Let $f$ be a meromorphic function and let $k \in \mathbb{N}$. Then

$$
m\left(r, \frac{f^{(k)}}{f}\right)=S(r, f),
$$


where $S(r, f)=O(\log T(r, f)+\log r)$, possibly outside a set $E_{9} \subset(0,+\infty)$ with a finite linear measure. If $f$ is of a finite order of growth, then

$$
m\left(r, \frac{f^{(k)}}{f}\right)=O(\log r) .
$$

Lemma 2.13. ([6]) Let $A_{0}, A_{1}, \ldots, A_{k-1}, F \not \equiv 0$ are meromorphic functions of a finite order. If $f$ is a meromorphic solution with $\rho(f)=+\infty$ of the equation

$$
f^{(k)}+A_{k-1} f^{(k-1)}+\cdots+A_{1} f^{\prime}+A_{0} f=F,
$$

then

$$
\bar{\lambda}(f)=\lambda(f)=\rho(f)=+\infty .
$$

\section{Proof of Theorem 1.5}

First, we prove that each meromorphic solution $f(\not \equiv 0)$ of the equation $(1.4)$ is transcendental of order $\rho(f) \geqslant n$. We assume that $f(\not \equiv 0)$ is a meromorphic solution of equation (1.4) with $\rho(f)<n$. We can rewrite equation $(1.4)$ as

$$
\begin{aligned}
\left(A_{k-1,1}(z) e^{P_{k-1}(z)}\right. & \left.+A_{k-1,2}(z) e^{Q_{k-1}(z)}\right) f^{(k-1)} \\
& +\cdots+\left(A_{0,1}(z) e^{P_{0}(z)}+A_{0,2}(z) e^{Q_{0}(z)}\right) f=-f^{(k)} .
\end{aligned}
$$

Since

$$
\max \left\{\rho\left(A_{j i}\right), j=0,1, \ldots, k-1 ; i=1,2\right\}<n
$$

and

$$
\rho(f)<n,
$$

then $A_{j i} f^{(j)}, j=0,1, \ldots, k-1 ; i=1,2$ and $f^{(k)}$ are meromorphic functions of a finite order with

$$
\rho\left(A_{j i} f^{(j)}\right)<n \text { and } \rho\left(f^{(k)}\right)<n .
$$

We have also $a_{0, n} \neq b_{0, n}$ and $a_{j, n}=c_{j} a_{0, n}, b_{j, n}=c_{j} b_{0, n}, c_{j}>1, j=1, \ldots, k-1$. Hence, $a_{j, n} \neq b_{j, n}$ and therefore $\operatorname{deg}\left(P_{j}-P_{0}\right)=\operatorname{deg}\left(Q_{j}-Q_{0}\right)=n$. Since $A_{0,1}(z) f \neq 0, A_{0,2}(z) f \neq 0$, by Lemma 2.1, we find that the order of growth of the left side of equation (3.1) is $n$, this contradicts the inequality $\rho\left(f^{(k)}\right)<n$. Thus, each meromorphic solution $f(\not \equiv 0)$ of equation (1.4) is transcendental with order $\rho(f) \geqslant n$.

Let $z=r e^{i \theta}, a_{0, n}=\left|a_{0, n}\right| e^{i \theta_{1}}, b_{0, n}=\left|b_{0, n}\right| e^{i \theta_{2}}, \theta_{1}, \theta_{2} \in[0,2 \pi)$. Then

$$
\delta\left(P_{0}, \theta\right)=\left|a_{0, n}\right| \cos \left(n \theta+\theta_{1}\right), \delta\left(Q_{0}, \theta\right)=\left|b_{0, n}\right| \cos \left(n \theta+\theta_{2}\right) .
$$

Since $a_{j, n}=c_{j} a_{0, n}, b_{j, n}=c_{j} b_{0, n}, c_{j}>1, j=1, \ldots, k-1$, and $c_{j}$ are distinct numbers, we have

$$
\delta\left(P_{j}, \theta\right)=c_{j} \delta\left(P_{0}, \theta\right), \delta\left(Q_{j}, \theta\right)=c_{j} \delta\left(Q_{0}, \theta\right),
$$

and there exists exactly one $c_{s}$ such that $c_{s}=\max \left\{c_{j}, j=0,1, \ldots, k-1\right\}$. Let $c_{0}=1$.

We split our proof into two cases: $\theta_{1}=\theta_{2}$ and $\theta_{1} \neq \theta_{2}$

Case 1. As $\theta_{1}=\theta_{2}$, because of $a_{0, n} \neq b_{0, n}$, we suppose $\left|a_{0, n}\right|<\left|b_{0, n}\right|$ without loss of generality. Assume that $f$ is a meromorphic solution to equation (1.4) with poles of uniformly bounded multiplicity. From (1.4), we have

$$
\begin{aligned}
\mid A_{s, 1}(z) e^{P_{s}(z)} & +A_{s, 2}(z) e^{Q_{s}(z)} \mid \\
& \leqslant\left|\frac{f}{f^{(s)}}\right|\left(\left|\frac{f^{(k)}}{f}\right|+\sum_{j=0, j \neq s}^{k-1}\left\{\left|A_{j, 1}(z) e^{P_{j}(z)}+A_{j, 2}(z) e^{Q_{j}(z)}\right|\left|\frac{f^{(j)}}{f}\right|\right\}\right) .
\end{aligned}
$$


Since $f$ is transcendental, then by Lemma 2.2 , for $\alpha=2$, there exist a set $E_{1} \subset(1,+\infty)$ with $m_{l}\left(E_{1}\right)<+\infty$ and a constant $B>0$ such that for all $z$ satisfying $|z|=r \notin[0,1] \cup E_{1}$, we have

$$
\left|\frac{f^{(j)}(z)}{f(z)}\right| \leqslant B[T(2 r, f)]^{k+1}, j=1,2, \ldots, k, j \neq s .
$$

By (1.4), it follows that the poles of $f$ can be located only at the poles of $A_{j i}(z)$, $j=0,1, \ldots, k-1 ; i=1,2$. We observe that the poles of $f$ are of uniformly bounded multiplicity. Hence,

$$
\lambda(1 / f) \leqslant \max \left\{\rho\left(A_{j i}\right), j=0,1, \ldots, k-1 ; i=1,2\right\}<n .
$$

By Hadamard factorization theorem, we know that $f$ can be expressed as $f(z)=\frac{g(z)}{d(z)}$, where $g(z)$ and $d(z)$ are entire functions with

$$
\lambda(d)=\rho(d)=\lambda(1 / f)<n \leqslant \rho(f)=\rho(g) .
$$

For each sufficiently large $|z|=r$, let $z_{r}=r e^{i \theta_{r}}$ be a point satisfying $\left|g\left(z_{r}\right)\right|=M(r, g)$. By Lemma 2.11, there exist a constant $\delta_{r}(>0)$, a sequence $\left\{r_{m}\right\}_{m \in \mathbb{N}}, r_{m} \rightarrow+\infty$ and a set $E_{8}$ of a finite logarithmic measure such that the estimate

$$
\left|\frac{f(z)}{f^{(s)}(z)}\right| \leqslant r_{m}^{2 s}
$$

holds for all $z$ satisfying $|z|=r_{m} \notin E_{8}, r_{m} \rightarrow+\infty$ and $\arg z=\theta \in\left[\theta_{r}-\delta_{r}, \theta_{r}+\delta_{r}\right]$.

(i) If $\delta\left(P_{0}, \theta\right)>0$, then by $(3.3)$ we have

$$
\delta\left(Q_{j}, \theta\right)>\delta\left(Q_{0}, \theta\right)>0, \quad \delta\left(Q_{j}, \theta\right)>\delta\left(P_{j}, \theta\right)>\delta\left(P_{0}, \theta\right)>0 .
$$

By Lemma 2.3, for any given $\varepsilon$ obeying

$$
0<\varepsilon<\min \left\{\frac{1}{2}\left(\frac{c_{s}-c_{j}}{c_{s}+c_{j}}\right), j \neq s\right\},
$$

there exists a set $E_{2} \subset[1,+\infty)$ of a finite logarithmic measure such that for all $z$ satisfying $|z|=r \notin[0,1] \cup E_{2}, r \rightarrow+\infty$ and $\arg z=\theta \in\left[\theta_{r}-\delta_{r}, \theta_{r}+\delta_{r}\right] \backslash H$, where

$$
H=\left\{\theta \in[0 ; 2 \pi): \delta\left(P_{0}, \theta\right)=0, \delta\left(Q_{0}, \theta\right)=0\right\}
$$

is a finite set, we have

$$
\begin{aligned}
&\left|A_{s, 1}(z) e^{P_{s}(z)}+A_{s, 2}(z) e^{Q_{s}(z)}\right| \geqslant\left|A_{s, 2}(z) e^{Q_{s}(z)}\right|-\left|A_{s, 1}(z) e^{P_{s}(z)}\right| \\
& \geqslant \exp \left\{(1-\varepsilon) c_{s} \delta\left(Q_{0}, \theta\right) r^{n}\right\}-\exp \left\{(1+\varepsilon) c_{s} \delta\left(P_{0}, \theta\right) r^{n}\right\} \\
& \geqslant \frac{1}{2} \exp \left\{(1-\varepsilon) c_{s} \delta\left(Q_{0}, \theta\right) r^{n}\right\} \\
&\left|A_{j, 1}(z) e^{P_{j}(z)}+A_{j, 2}(z) e^{Q_{j}(z)}\right| \leqslant\left|A_{j, 1}(z) e^{P_{j}(z)}\right|+\left|A_{j, 2}(z) e^{Q_{j}(z)}\right| \\
& \leqslant \exp \left\{(1+\varepsilon) c_{j} \delta\left(P_{0}, \theta\right) r^{n}\right\}+\exp \left\{(1+\varepsilon) c_{j} \delta\left(Q_{0}, \theta\right) r^{n}\right\} \\
& \leqslant 2 \exp \left\{(1+\varepsilon) c_{j} \delta\left(Q_{0}, \theta\right) r^{n}\right\}, j=0,1,2, \ldots, k-1, j \neq s .
\end{aligned}
$$

Substituting (3.5), (3.6), (3.7), 3.8) into (3.4), for all $z$ satisfying $|z|=r_{m} \notin[0,1] \cup E_{1} \cup E_{2} \cup E_{8}$, $r_{m} \rightarrow+\infty$ and $\arg z=\theta \in\left[\theta_{r}-\delta_{r}, \theta_{r}+\delta_{r}\right] \backslash H$ we obtain

$$
\begin{aligned}
\frac{1}{2} \exp \left\{(1-\varepsilon) c_{s} \delta\left(Q_{0}, \theta\right) r_{m}^{n}\right\} \leqslant & r_{m}^{2 s}\left(B\left[T\left(2 r_{m}, f\right)\right]^{k+1}\right. \\
& \left.+B\left[T\left(2 r_{m}, f\right)\right]^{k+1} \sum_{j=0, j \neq s}^{k-1} 2 \exp \left\{(1+\varepsilon) c_{j} \delta\left(Q_{0}, \theta\right) r_{m}^{n}\right\}\right)
\end{aligned}
$$




$$
\leqslant 4 r_{m}^{2 s} B\left[T\left(2 r_{m}, f\right)\right]^{k+1} \sum_{j=0, j \neq s}^{k-1} \exp \left\{(1+\varepsilon) c_{j} \delta\left(Q_{0}, \theta\right) r_{m}^{n}\right\}
$$

which gives

$$
\exp \left\{(1-\varepsilon) c_{s} \delta\left(Q_{0}, \theta\right) r_{m}^{n}\right\} \leqslant 8 r_{m}^{2 s} B\left[T\left(2 r_{m}, f\right)\right]^{k+1} \sum_{j=0, j \neq s}^{k-1} \exp \left\{(1+\varepsilon) c_{j} \delta\left(Q_{0}, \theta\right) r_{m}^{n}\right\} .
$$

Since $0<\varepsilon<\min \left\{\frac{1}{2}\left(\frac{c_{s}-c_{j}}{c_{s}+c_{j}}\right), j \neq s\right\}$, then by Lemma 2.6 and 3.9 we obtain

$$
\rho(f)=\limsup _{r_{m} \rightarrow+\infty} \frac{\log T\left(r_{m}, f\right)}{\log r_{m}}=+\infty,
$$

and

$$
\rho_{2}(f)=\limsup _{r_{m} \rightarrow+\infty} \frac{\log \log T\left(r_{m}, f\right)}{\log r_{m}} \geqslant n .
$$

In addition, by Lemma 2.7 and from equation 1.4 , we have $\rho_{2}(f) \leqslant n$, so $\rho_{2}(f)=n$.

(ii) If $\delta\left(P_{0}, \theta\right)<0$, then by $(3.2)$ and $(3.3)$ we have

$$
\delta\left(Q_{j}, \theta\right)<\delta\left(Q_{0}, \theta\right)<\delta\left(P_{0}, \theta\right)<0, \quad \delta\left(P_{j}, \theta\right)<\delta\left(P_{0}, \theta\right)<0 .
$$

By Lemma 2.3. for any given $0<\varepsilon<1$, there exists a set $E_{2} \subset[1,+\infty)$ of a finite logarithmic measure such that for all $z$ satisfying $|z|=r \notin[0,1] \cup E_{2}, r \rightarrow+\infty$ and $\arg z=\theta \in\left[\theta_{r}-\delta_{r}, \theta_{r}+\delta_{r}\right] \backslash H$, where $H=\left\{\theta \in[0 ; 2 \pi): \delta\left(P_{0}, \theta\right)=0, \delta\left(Q_{0}, \theta\right)=0\right\}$ is a finite set, we get

$$
\begin{aligned}
\left|A_{j, 1}(z) e^{P_{j}(z)}+A_{j, 2}(z) e^{Q_{j}(z)}\right| & \leqslant\left|A_{j, 1}(z) e^{P_{j}(z)}\right|+\left|A_{j, 2}(z) e^{Q_{j}(z)}\right| \\
& \leqslant \exp \left\{(1-\varepsilon) \delta\left(P_{j}, \theta\right) r^{n}\right\}+\exp \left\{(1-\varepsilon) \delta\left(Q_{j}, \theta\right) r^{n}\right\} \\
& \leqslant 2 \exp \left\{(1-\varepsilon) \delta\left(P_{0}, \theta\right) r^{n}\right\}, \quad j=0,1,2, \ldots, k-1 .
\end{aligned}
$$

By (1.4) we have

$$
1 \leqslant\left|\frac{f}{f^{(k)}}\right| \sum_{j=0}^{k-1}\left\{\left|A_{j, 1}(z) e^{P_{j}(z)}+A_{j, 2}(z) e^{Q_{j}(z)}\right|\left|\frac{f^{(j)}}{f}\right|\right\} .
$$

Substituting (3.5), (3.6) and (3.10) into (3.11), for all $z$ satisfying $|z|=r_{m} \notin[0,1] \cup E_{1} \cup E_{2} \cup E_{8}$, $r_{m} \rightarrow+\infty$ and $\arg z=\theta \in\left[\theta_{r}-\delta_{r}, \theta_{r}+\delta_{r}\right] \backslash H$ we obtain

$$
\begin{aligned}
1 & \leqslant r_{m}^{2 k} B\left[T\left(2 r_{m}, f\right)\right]^{k+1}\left(\sum_{j=0}^{k-1} 2 \exp \left\{(1-\varepsilon) \delta\left(P_{0}, \theta\right) r_{m}^{n}\right\}\right) \\
& \leqslant 2 k r_{m}^{2 k} B\left[T\left(2 r_{m}, f\right)\right]^{k+1} \exp \left\{(1-\varepsilon) \delta\left(P_{0}, \theta\right) r_{m}^{n}\right\}
\end{aligned}
$$

which gives

$$
\exp \left\{(\varepsilon-1) \delta\left(P_{0}, \theta\right) r_{m}^{n}\right\} \leqslant 2 k r_{m}^{2 k} B\left[T\left(2 r_{m}, f\right)\right]^{k+1} .
$$

By Lemma 2.6 and (3.13) we obtain

$$
\rho(f)=\limsup _{r_{m} \rightarrow+\infty} \frac{\log ^{+} T\left(r_{m}, f\right)}{\log r_{m}}=+\infty
$$

and

$$
\rho_{2}(f)=\limsup _{r \rightarrow+\infty} \frac{\log _{2}^{+} T\left(r_{m}, f\right)}{\log r_{m}} \geqslant n .
$$

In addition, by Lemma 2.7 and equation 1.4 , we have $\rho_{2}(f) \leqslant n$, so $\rho_{2}(f)=n$.

Case 2 Assume that $\theta_{1} \neq \theta_{2}$. 
(i) If $\delta\left(P_{0}, \theta\right)>0, \delta\left(Q_{0}, \theta\right)<0$, then by $(3.3)$, we get

$$
\delta\left(P_{j}, \theta\right)>\delta\left(P_{0}, \theta\right)>0, \quad \delta\left(Q_{j}, \theta\right)<\delta\left(Q_{0}, \theta\right)<0,
$$

by Lemma 2.3 , for any given $0<\varepsilon<\min \left\{\frac{1}{2}\left(\frac{c_{s}-c_{j}}{c_{s}+c_{j}}\right), j \neq s\right\}$, there exists a set $E_{2} \subset[1,+\infty)$ of a finite logarithmic measure such that for all $z$ satisfying $|z|=r \notin[0,1] \cup E_{2}, r \rightarrow+\infty$ and $\arg z=\theta \in\left[\theta_{r}-\delta_{r}, \theta_{r}+\delta_{r}\right] \backslash H_{1}$, where

$$
H_{1}=\left\{\theta \in[0,2 \pi): \delta\left(P_{0}, \theta\right)=0, \delta\left(Q_{0}, \theta\right)=0, \delta\left(P_{0}, \theta\right)=\delta\left(Q_{0}, \theta\right)\right\}
$$

is a finite set, we have

$$
\begin{aligned}
\mid A_{s, 1}(z) e^{P_{s}(z)} & +A_{s, 2}(z) e^{Q_{s}(z)}|\geqslant| A_{s, 1}(z) e^{P_{s}(z)}|-| A_{s, 2}(z) e^{Q_{s}(z)} \mid \\
& \geqslant \exp \left\{(1-\varepsilon) c_{s} \delta\left(P_{0}, \theta\right) r^{n}\right\}-\exp \left\{(1-\varepsilon) c_{s} \delta\left(Q_{0}, \theta\right) r^{n}\right\} \\
& \geqslant \frac{1}{2} \exp \left\{(1-\varepsilon) c_{s} \delta\left(P_{0}, \theta\right) r^{n}\right\}, \\
\mid A_{j, 1}(z) e^{P_{j}(z)} & +A_{j, 2}(z) e^{Q_{j}(z)}|\leqslant| A_{j, 1}(z) e^{P_{j}(z)}|+| A_{j, 2}(z) e^{Q_{j}(z)} \mid \\
& \leqslant \exp \left\{(1+\varepsilon) c_{j} \delta\left(P_{0}, \theta\right) r^{n}\right\}+\exp \left\{(1-\varepsilon) c_{j} \delta\left(Q_{0}, \theta\right) r^{n}\right\} \\
& \leqslant 2 \exp \left\{(1+\varepsilon) c_{j} \delta\left(P_{0}, \theta\right) r^{n}\right\}, j=0,1,2, \ldots, k-1, \quad j \neq s .
\end{aligned}
$$

By (3.4), (3.5), (3.6), (3.14) and (3.15), for all $z$ satisfying $|z|=r_{m} \notin[0,1] \cup E_{1} \cup E_{2} \cup E_{8}$, $r_{m} \rightarrow+\infty$ and $\arg z=\theta \in\left[\theta_{r}-\delta_{r}, \theta_{r}+\delta_{r}\right] \backslash H_{1}$ we have

$$
\begin{aligned}
\frac{1}{2} \exp \left\{(1-\varepsilon) c_{s} \delta\left(P_{0}, \theta\right) r_{m}^{n}\right\} \leqslant & r_{m}^{2 s}\left(B\left[T\left(2 r_{m}, f\right)\right]^{k+1}\right. \\
& \left.+B\left[T\left(2 r_{m}, f\right)\right]^{k+1} \sum_{j=0, j \neq s}^{k-1} 2 \exp \left\{(1+\varepsilon) c_{j} \delta\left(P_{0}, \theta\right) r_{m}^{n}\right\}\right) \\
\leqslant & 4 r_{m}^{2 s} B\left[T\left(2 r_{m}, f\right)\right]^{k+1} \sum_{j=0, j \neq s}^{k-1} \exp \left\{(1+\varepsilon) c_{j} \delta\left(P_{0}, \theta\right) r_{m}^{n}\right\}
\end{aligned}
$$

which gives

$$
\exp \left\{(1-\varepsilon) c_{s} \delta\left(P_{0}, \theta\right) r_{m}^{n}\right\} \leqslant 8 r_{m}^{2 s} B\left[T\left(2 r_{m}, f\right)\right]^{k+1} \sum_{j=0, j \neq s}^{k-1} \exp \left\{(1+\varepsilon) c_{j} \delta\left(P_{0}, \theta\right) r_{m}^{n}\right\}
$$

Since $0<\varepsilon<\min \left\{\frac{1}{2}\left(\frac{c_{s}-c_{j}}{c_{s}+c_{j}}\right), j \neq s\right\}$, then by Lemma 2.6 and 3.16 we obtain

$$
\rho(f)=\limsup _{r_{m} \rightarrow+\infty} \frac{\log T\left(r_{m}, f\right)}{\log r_{m}}=+\infty
$$

and

$$
\rho_{2}(f)=\limsup _{r_{m} \rightarrow+\infty} \frac{\log \log T\left(r_{m}, f\right)}{\log r_{m}} \geqslant n .
$$

In addition, by Lemma 2.7 and from equation $(1.4)$, we have $\rho_{2}(f) \leqslant n$, so $\rho_{2}(f)=n$.

(ii) If $\delta\left(P_{0}, \theta\right)<0, \delta\left(Q_{0}, \theta\right)>0$, by (3.3), we have

$$
\delta\left(P_{j}, \theta\right)<\delta\left(P_{0}, \theta\right)<0, \quad \delta\left(Q_{j}, \theta\right)>\delta\left(Q_{0}, \theta\right)>0 .
$$

By Lemma 2.3. for any given $0<\varepsilon<\min \left\{\frac{1}{2}\left(\frac{c_{s}-c_{j}}{c_{s}+c_{j}}\right), j \neq s\right\}$, there exists a set $E_{2} \subset[1,+\infty)$ of a finite logarithmic measure such that for all $z$ satisfying $|z|=r \notin[0,1] \cup E_{2}, r \rightarrow+\infty$ and $\arg z=\theta \in\left[\theta_{r}-\delta_{r}, \theta_{r}+\delta_{r}\right] \backslash H_{1}$, where

$$
H_{1}=\left\{\theta \in[0,2 \pi): \delta\left(P_{0}, \theta\right)=0, \delta\left(Q_{0}, \theta\right)=0, \delta\left(P_{0}, \theta\right)=\delta\left(Q_{0}, \theta\right)\right\}
$$


is a finite set, we have

$$
\begin{aligned}
\mid A_{s, 1}(z) e^{P_{s}(z)} & +A_{s, 2}(z) e^{Q_{s}(z)}|\geqslant| A_{s, 2}(z) e^{Q_{s}(z)}|-| A_{s, 1}(z) e^{P_{s}(z)} \mid \\
& \geqslant \exp \left\{(1-\varepsilon) c_{s} \delta\left(Q_{0}, \theta\right) r^{n}\right\}-\exp \left\{(1-\varepsilon) c_{s} \delta\left(P_{0}, \theta\right) r^{n}\right\} \\
& \geqslant \frac{1}{2} \exp \left\{(1-\varepsilon) c_{s} \delta\left(Q_{0}, \theta\right) r^{n}\right\} \\
\mid A_{j, 1}(z) e^{P_{j}(z)} & +A_{j, 2}(z) e^{Q_{j}(z)}|\leqslant| A_{j, 1}(z) e^{P_{j}(z)}|+| A_{j, 2}(z) e^{Q_{j}(z)} \mid \\
& \leqslant \exp \left\{(1+\varepsilon) c_{j} \delta\left(_{0}, \theta\right) r^{n}\right\}+\exp \left\{(1-\varepsilon) c_{j} \delta\left(P_{0}, \theta\right) r^{n}\right\} \\
& \leqslant 2 \exp \left\{(1+\varepsilon) c_{j} \delta\left(Q_{0}, \theta\right) r^{n}\right\}, j=0,1,2, \ldots, k-1, j \neq s .
\end{aligned}
$$

Proceeding as in the proof of (i), for all $z$ satisfying $|z|=r_{m} \notin[0,1] \cup E_{1} \cup E_{2} \cup E_{8}, r_{m} \rightarrow+\infty$ and $\arg z=\theta \in\left[\theta_{r}-\delta_{r}, \theta_{r}+\delta_{r}\right] \backslash H_{1}$ we obtain

$$
\begin{aligned}
\frac{1}{2} \exp \left\{(1-\varepsilon) c_{s} \delta\left(Q_{0}, \theta\right) r_{m}^{n}\right\} & \leqslant r_{m}^{2 s}\left(B\left[T\left(2 r_{m}, f\right)\right]^{k+1}\right. \\
& \left.+B\left[T\left(2 r_{m}, f\right)\right]^{k+1} \sum_{j=0, j \neq s}^{k-1} 2 \exp \left\{(1+\varepsilon) c_{j} \delta\left(Q_{0}, \theta\right) r_{m}^{n}\right\}\right) \\
\leqslant & 4 r_{m}^{2 s} B\left[T\left(2 r_{m}, f\right)\right]^{k+1} \sum_{j=0, j \neq s}^{k-1} \exp \left\{(1+\varepsilon) c_{j} \delta\left(Q_{0}, \theta\right) r_{m}^{n}\right\},
\end{aligned}
$$

which gives

$$
\exp \left\{(1-\varepsilon) c_{s} \delta\left(Q_{0}, \theta\right) r_{m}^{n}\right\} \leqslant 8 r_{m}^{2 s} B\left[T\left(2 r_{m}, f\right)\right]^{k+1} \sum_{j=0, j \neq s}^{k-1} \exp \left\{(1+\varepsilon) c_{j} \delta\left(Q_{0}, \theta\right) r_{m}^{n}\right\} \text {. }
$$

Since $0<\varepsilon<\min \left\{\frac{1}{2}\left(\frac{c_{s}-c_{j}}{c_{s}+c_{j}}\right), j \neq s\right\}$, then by Lemma 2.6 and 3.19 we obtain

$$
\rho(f)=\limsup _{r_{m} \rightarrow+\infty} \frac{\log T\left(r_{m}, f\right)}{\log r_{m}}=+\infty
$$

and

$$
\rho_{2}(f)=\limsup _{r_{m} \rightarrow+\infty} \frac{\log \log T\left(r_{m}, f\right)}{\log r_{m}} \geqslant n .
$$

In addition, by Lemma 2.7 and from equation 1.4 , we have $\rho_{2}(f) \leqslant n$, so $\rho_{2}(f)=n$.

(iii) If $\delta\left(P_{0}, \theta\right)>0, \delta\left(Q_{0}, \theta\right)>0$, then by $(3.3)$, we have

$$
\delta\left(P_{j}, \theta\right)>\delta\left(P_{0}, \theta\right)>0, \delta\left(Q_{j}, \theta\right)>\delta\left(Q_{0}, \theta\right)>0 .
$$

We suppose $\delta\left(P_{0}, \theta\right)>\delta\left(Q_{0}, \theta\right)$ without loss of generality. By Lemma 2.3, for any given $0<\varepsilon<\min \left\{\frac{1}{2}\left(\frac{c_{s}-c_{j}}{c_{s}+c_{j}}\right), j \neq s\right\}$, there exists a set $E_{2} \subset[1,+\infty)$ of a finite logarithmic measure such that for all $z$ satisfying $|z|=r \notin[0,1] \cup E_{2}, r \rightarrow+\infty$ and $\arg z=\theta \in\left[\theta_{r}-\delta_{r}, \theta_{r}+\delta_{r}\right] \backslash H_{1}$, where

$$
H_{1}=\left\{\theta \in[0,2 \pi): \delta\left(P_{0}, \theta\right)=0, \delta\left(Q_{0}, \theta\right)=0, \delta\left(P_{0}, \theta\right)=\delta\left(Q_{0}, \theta\right)\right\}
$$

is a finite set, we have

$$
\begin{aligned}
\mid A_{s, 1}(z) e^{P_{s}(z)} & +A_{s, 2}(z) e^{Q_{s}(z)}|\geqslant| A_{s, 1}(z) e^{P_{s}(z)}|-| A_{s, 2}(z) e^{Q_{s}(z)} \mid \\
& \geqslant \exp \left\{(1-\varepsilon) c_{s} \delta\left(P_{0}, \theta\right) r^{n}\right\}-\exp \left\{(1-\varepsilon) c_{s} \delta\left(Q_{0}, \theta\right) r^{n}\right\} \\
& \geqslant \frac{1}{2} \exp \left\{(1-\varepsilon) c_{s} \delta\left(P_{0}, \theta\right) r^{n}\right\}
\end{aligned}
$$




$$
\begin{aligned}
\mid A_{j, 1}(z) e^{P_{j}(z)} & +A_{j, 2}(z) e^{Q_{j}(z)}|\leqslant| A_{j, 1}(z) e^{P_{j}(z)}|+| A_{j, 2}(z) e^{Q_{j}(z)} \mid \\
& \leqslant \exp \left\{(1+\varepsilon) c_{j} \delta\left(P_{0}, \theta\right) r^{n}\right\}+\exp \left\{(1+\varepsilon) c_{j} \delta\left(Q_{0}, \theta\right) r^{n}\right\} \\
& \leqslant 2 \exp \left\{(1+\varepsilon) c_{j} \delta\left(P_{0}, \theta\right) r^{n}\right\}, j=0,1,2, \ldots, k-1, j \neq s .
\end{aligned}
$$

From (3.4), (3.5), (3.6), (3.20) and (3.21), we have for all $z$ satisfying $|z|=r_{m} \notin[0,1] \cup E_{1} \cup$ $E_{2} \cup E_{8}, r_{m} \rightarrow+\infty$ and $\arg z=\theta \in\left[\theta_{r}-\delta_{r}, \theta_{r}+\delta_{r}\right] \backslash H_{1}$

$$
\frac{1}{2} \exp \left\{(1-\varepsilon) c_{s} \delta\left(P_{0}, \theta\right) r_{m}^{n}\right\} \leqslant 4 r_{m}^{2 s} B\left[T\left(2 r_{m}, f\right)\right]^{k+1} \sum_{j=0, j \neq s}^{k-1} \exp \left\{(1+\varepsilon) c_{j} \delta\left(P_{0}, \theta\right) r_{m}^{n}\right\},
$$

which gives

$$
\exp \left\{(1-\varepsilon) c_{s} \delta\left(P_{0}, \theta\right) r_{m}^{n}\right\} \leqslant 8 r_{m}^{2 s} B\left[T\left(2 r_{m}, f\right)\right]^{k+1} \sum_{j=0, j \neq s}^{k-1} \exp \left\{(1+\varepsilon) c_{j} \delta\left(P_{0}, \theta\right) r_{m}^{n}\right\} \text {. }
$$

Since $0<\varepsilon<\min \left\{\frac{1}{2}\left(\frac{c_{s}-c_{j}}{c_{s}+c_{j}}\right), j \neq s\right\}$, then by Lemma 2.6 and 3.22 we obtain

$$
\rho(f)=\limsup _{r_{m} \rightarrow+\infty} \frac{\log T\left(r_{m}, f\right)}{\log r_{m}}=+\infty,
$$

and

$$
\rho_{2}(f)=\limsup _{r_{m} \rightarrow+\infty} \frac{\log \log T\left(r_{m}, f\right)}{\log r_{m}} \geqslant n .
$$

In addition, by Lemma 2.7 and from equation 1.4 , we have $\rho_{2}(f) \leqslant n$, so $\rho_{2}(f)=n$.

(iv) If $\delta\left(P_{0}, \theta\right)<0, \delta\left(Q_{0}, \theta\right)<0$, then by (3.3), we have

$$
\delta\left(P_{j}, \theta\right)<\delta\left(P_{0}, \theta\right)<0, \delta\left(Q_{j}, \theta\right)<\delta\left(Q_{0}, \theta\right)<0 .
$$

Let $\delta=\max \left\{\delta\left(P_{0}, \theta\right), \delta\left(Q_{0}, \theta\right)\right\}$. Then, by Lemma 2.3, for any given $0<\varepsilon<1$, there exists a set $E_{2} \subset[1,+\infty)$ of a finite logarithmic measure such that for all $z$ satisfying $|z|=r \notin[0,1] \cup E_{2}$, $r \rightarrow+\infty$ and $\arg z=\theta \in\left[\theta_{r}-\delta_{r}, \theta_{r}+\delta_{r}\right] \backslash H_{1}$, where

$$
H_{1}=\left\{\theta \in[0,2 \pi): \delta\left(P_{0}, \theta\right)=0, \delta\left(Q_{0}, \theta\right)=0, \delta\left(P_{0}, \theta\right)=\delta\left(Q_{0}, \theta\right)\right\}
$$

is a finite set, we get

$$
\begin{aligned}
\left|A_{j, 1}(z) e^{P_{j}(z)}+A_{j, 2}(z) e^{Q_{j}(z)}\right| & \leqslant\left|A_{j, 1}(z) e^{P_{j}(z)}\right|+\left|A_{j, 2}(z) e^{Q_{j}(z)}\right| \\
& \leqslant \exp \left\{(1-\varepsilon) c_{j} \delta\left(P_{0}, \theta\right) r^{n}\right\}+\exp \left\{(1-\varepsilon) c_{j} \delta\left(Q_{0}, \theta\right) r^{n}\right\} \\
& \leqslant 2 \exp \left\{(1-\varepsilon) c_{j} \delta r^{n}\right\}, \quad j=0,1, \ldots, k-1 .
\end{aligned}
$$

By (3.5), (3.6), (3.11) and (3.23) for all $z$ satisfying $|z|=r_{m} \notin[0,1] \cup E_{1} \cup E_{2} \cup E_{8}, r_{m} \rightarrow+\infty$ and $\arg z=\theta \in\left[\theta_{r}-\delta_{r}, \theta_{r}+\delta_{r}\right] \backslash H_{1}$ we have

$$
\begin{aligned}
1 & \leqslant r_{m}^{2 k} B\left[T\left(2 r_{m}, f\right)\right]^{k+1}\left\{\sum_{j=0}^{k-1} 2 \exp \left\{(1-\varepsilon) c_{j} \delta r_{m}^{n}\right\}\right\} \\
& \leqslant 2 r_{m}^{2 k} B\left[T\left(2 r_{m}, f\right)\right]^{k+1}\left\{\sum_{j=0}^{k-1} \exp \left\{(1-\varepsilon) c_{j} \delta r_{m}^{n}\right\}\right\} .
\end{aligned}
$$

Since $c_{j}>1, j=1, \ldots, k-1$ and $\delta<0$, we obtain

$$
\exp \left\{(1-\varepsilon) c_{j} \delta r_{m}^{n}\right\} \leqslant \exp \left\{(1-\varepsilon) \delta r_{m}^{n}\right\}, \quad j=1, \ldots, k-1
$$

so 3.24 becomes

$$
1 \leqslant 2 r_{m}^{2 k} k B\left[T\left(2 r_{m}, f\right)\right]^{k+1} \exp \left\{(1-\varepsilon) \delta r_{m}^{n}\right\}
$$


which gives

$$
\exp \left\{(\varepsilon-1) \delta r_{m}^{n}\right\} \leqslant 2 r_{m}^{2 k} B k\left[T\left(2 r_{m}, f\right)\right]^{k+1} .
$$

By Lemma 2.6 and 3.25 we obtain

$$
\rho(f)=\limsup _{r_{m} \rightarrow+\infty} \frac{\log T\left(r_{m}, f\right)}{\log r_{m}}=+\infty
$$

and

$$
\rho_{2}(f)=\limsup _{r_{m} \rightarrow+\infty} \frac{\log \log T\left(r_{m}, f\right)}{\log r_{m}} \geqslant n .
$$

In addition, by Lemma 2.7 and from equation 1.4 , we have $\rho_{2}(f) \leqslant n$, so $\rho_{2}(f)=n$. This completes the proof of Theorem 1.5 .

\section{Proof of Theorem 1.6}

(i) Suppose $f_{0}$ is a meromorphic solution of a finite order to equation 1.5 with poles of uniformly bounded multiplicities. If $f_{1}\left(\not \equiv f_{0}\right)$ is an another meromorphic solution of a finite order to equation (1.5) with poles of uniformly bounded multiplicities, the function $f_{1}-f_{0}$ is a nonzero meromorphic solution to equation (1.4) with $\rho\left(f_{1}-f_{0}\right)<+\infty$. This contradicts Theorem 1.5. Hence, equation (1.5) has at most one meromorphic solution of a finite order. We assume that $f(z)$ is a meromorphic solution of infinite order to 1.5 with poles of uniformly bounded multiplicity. By (1.5), it is easy to see that if $f$ has a zero of order $\alpha(\alpha>k)$ at $z_{0}$, and $B_{0}, B_{1}, \ldots, B_{k-1}$ are analytic at $z_{0}$, then $F$ must have a zero at $z_{0}$ of order at least $\alpha-k$. Hence,

$$
n\left(r, \frac{1}{f}\right) \leqslant k \bar{n}\left(r, \frac{1}{f}\right)+n\left(r, \frac{1}{F}\right)+\sum_{j=0}^{k-1} n\left(r, B_{j}\right)
$$

and

$$
N\left(r, \frac{1}{f}\right) \leqslant k \bar{N}\left(r, \frac{1}{f}\right)+N\left(r, \frac{1}{F}\right)+\sum_{j=0}^{k-1} N\left(r, B_{j}\right)
$$

where $B_{j}(z)=A_{j 1}(z) e^{P_{j}(z)}+A_{j 2}(z) e^{Q_{j}(z)}, j=0,1,2, \ldots, k-1$. Now (1.5) can be rewritten as

$$
\frac{1}{f}=\frac{1}{F}\left(\frac{f^{(k)}}{f}+B_{k-1}(z) \frac{f^{(k-1)}}{f}+\cdots+B_{1}(z) \frac{f^{\prime}}{f}+B_{0}(z)\right) .
$$

By Lemma 2.12 and $(4.2)$, we get for $|z|=r$ outside a set $E_{9}$ of finite linear measure, we have

$$
\begin{aligned}
m\left(r, \frac{1}{f}\right) & \leqslant m\left(r, \frac{1}{F}\right)+\sum_{j=1}^{k} m\left(r, \frac{f^{(j)}}{f}\right)+\sum_{j=0}^{k-1} m\left(r, B_{j}\right)+O(1) \\
& \leqslant m\left(r, \frac{1}{F}\right)+\sum_{j=0}^{k-1} m\left(r, B_{j}\right)+O(\log r T(r, f)) .
\end{aligned}
$$

Therefore, by 4.1), 4.3) and the first main theorem, there holds

$$
T(r, f)=T\left(r, \frac{1}{f}\right)+O(1) \leqslant T(r, F)+\sum_{j=0}^{k-1} T\left(r, B_{j}\right)+k \bar{N}\left(r, \frac{1}{f}\right)+O(\log r T(r, f))
$$

for all sufficiently large $r \notin E_{9}$. For sufficiently large $r$, we have

$$
O(\log r T(r, f)) \leqslant \frac{1}{2} T(r, f) .
$$


Let $\rho_{1}=\max \{n, \rho(F)\}$. By Lemma 2.4, for any given $\varepsilon>0$, there exists a set $E_{3} \subset(1,+\infty)$ of a finite logarithmic measure such that

$$
T(r, F) \leqslant r^{\rho_{1}+\varepsilon}, T\left(r, B_{j}\right) \leqslant r^{\rho_{1}+\varepsilon}, j=0,1, \ldots, k-1,
$$

when $|z|=r \notin[0,1] \cup E_{3}, r \rightarrow+\infty$. By 4.4), 4.5) and (4.6), for $r \notin[0,1] \cup E_{3} \cup E_{9}$ sufficiently large, we obtain

$$
T(r, f) \leqslant r^{\rho_{1}+\varepsilon}+k r^{\rho_{1}+\varepsilon}+k \bar{N}\left(r, \frac{1}{f}\right)+\frac{1}{2} T(r, f)
$$

which gives

$$
T(r, f) \leqslant 2(k+1) r^{\rho_{1}+\varepsilon}+2 k \bar{N}\left(r, \frac{1}{f}\right)
$$

Hence,

$$
\rho_{2}(f) \leqslant \bar{\lambda}_{2}(f)
$$

and therefore,

$$
\rho_{2}(f) \leqslant \bar{\lambda}_{2}(f) \leqslant \lambda_{2}(f) .
$$

Since by the definition we have $\bar{\lambda}_{2}(f) \leqslant \lambda_{2}(f) \leqslant \rho_{2}(f)$, we get

$$
\bar{\lambda}_{2}(f)=\lambda_{2}(f)=\rho_{2}(f) .
$$

On the other hand, $\max \left\{\rho\left(A_{j i}\right), j=0,1, \ldots, k-1 ; i=1,2\right\}<n$ and $\rho\left(B_{j}\right)<+\infty$ for all $j=0,1, \ldots, k-1$, and $f(z)$ is a solution to 1.5 of infinite order. Hence, by Lemma 2.13 we obtain $\bar{\lambda}(f)=\lambda(f)=\rho(f)=+\infty$. Since $\rho\left(B_{j}\right) \leqslant n$, by Lemma 2.7, we have $\rho_{2}(f) \leqslant \max \{n, \rho(F)\}$.

(ii) Suppose $f_{0}$ is a meromorphic solution of the equation 1.5 with finite order, by Lemma 2.12, we have $m\left(r, \frac{f_{0}^{(j)}}{f_{0}}\right)=O(\log r), j=1, \ldots, k-1$. Using 4.2 , we can get for $|z|=r$ outside a set $E_{9}$ of finite linear measure, we have

$$
\begin{aligned}
m\left(r, \frac{1}{f_{0}}\right) & \leqslant m\left(r, \frac{1}{F}\right)+\sum_{j=1}^{k} m\left(r, \frac{f_{0}^{(j)}}{f_{0}}\right)+\sum_{j=0}^{k-1} m\left(r, B_{j}\right)+O(1) \\
& \leqslant m\left(r, \frac{1}{F}\right)+\sum_{j=0}^{k-1} m\left(r, B_{j}\right)+O(\log r)
\end{aligned}
$$

and

$$
N\left(r, \frac{1}{f_{0}}\right) \leqslant k \bar{N}\left(r, \frac{1}{f_{0}}\right)+N\left(r, \frac{1}{F}\right)+\sum_{j=0}^{k-1} N\left(r, B_{j}\right) .
$$

By 4.8 and 4.9, we get

$$
T\left(r, f_{0}\right)=T\left(r, \frac{1}{f_{0}}\right)+O(1) \leqslant T\left(r, \frac{1}{F}\right)+\sum_{j=0}^{k-1} T\left(r, B_{j}\right)+k \bar{N}\left(r, \frac{1}{f_{0}}\right)+O(\log r) .
$$

By 4.6 and 4.10, we get

$$
T\left(r, f_{0}\right) \leqslant(k+1) r^{\rho_{1}+\varepsilon}+k \bar{N}\left(r, \frac{1}{f_{0}}\right)+O(\log r) .
$$

Hence, we obtain

$$
\rho\left(f_{0}\right) \leqslant \max \left\{\bar{\lambda}\left(f_{0}\right), \rho_{1}\right\}=\max \left\{n, \bar{\lambda}\left(f_{0}\right), \rho(F)\right\} .
$$


(iii) First we prove that each meromorphic solution $f$ to equation 1.5 is transcendental of order $\rho(f) \geqslant n$. We assume that $f$ is a meromorphic solution to equation $(1.5)$ with $\rho(f)<n$. We can rewrite equation $(1.5)$ as

$$
\left(A_{k-1,1}(z) e^{P_{k-1}(z)}+A_{k-1,2}(z) e^{Q_{k-1}(z)}\right) f^{(k-1)}+\cdots+\left(A_{0,1}(z) e^{P_{0}(z)}+A_{0,2}(z) e^{Q_{0}(z)}\right) f=B(z),
$$

where

$$
B(z)=F(z)-f^{(k)} .
$$

Since $\max \left\{\rho\left(A_{j i}\right), j=0,1, \ldots, k-1 ; i=1,2, \rho(F)\right\}<n$ and $\rho(f)<n$, then $A_{j i} f^{(j)}$, $j=0,1, \ldots, k-1, i=1,2$, and $B(z)$ are meromorphic functions of a finite order with $\rho\left(A_{j i} f^{(j)}\right)<n$ and $\rho(B)<n$. We also have $a_{0, n} \neq b_{0, n}$ and $a_{j, n}=c_{j} a_{0, n}, b_{j, n}=c_{j} b_{0, n}$, $c_{j}>1, j=1, \ldots, k-1$, . Hence, $a_{j, n} \neq b_{j, n}$ and $\operatorname{deg}\left(P_{j}-P_{0}\right)=\operatorname{deg}\left(Q_{j}-Q_{0}\right)=n$. Since $A_{0,1}(z) f \not \equiv 0, A_{0,2}(z) f \not \equiv 0$, by Lemma 2.1 we find that the order of growth of the left hand side of equation (4.11) is $n$. This contradicts the inequality $\rho(B)<n$. Therefore, each meromorphic solution $f$ to equation (1.5) is transcendental and is of order $\rho(f) \geqslant n$.

Let $z=r e^{i \theta}, a_{0, n}=\left|a_{0, n}\right| e^{i \theta_{1}}, b_{0, n}=\left|b_{0, n}\right| e^{i \theta_{2}}, \theta_{1}, \theta_{2} \in[0,2 \pi)$. Then

$$
\delta\left(P_{0}, \theta\right)=\left|a_{0, n}\right| \cos \left(n \theta+\theta_{1}\right), \delta\left(Q_{0}, \theta\right)=\left|b_{0, n}\right| \cos \left(n \theta+\theta_{2}\right) .
$$

Since $a_{j, n}=c_{j} a_{0, n}, b_{j, n}=c_{j} b_{0, n}, c_{j}>1, j=1, \ldots, k-1$, and $c_{j}$ are distinct numbers, we have

$$
\delta\left(P_{j}, \theta\right)=c_{j} \delta\left(P_{0}, \theta\right), \delta\left(Q_{j}, \theta\right)=c_{j} \delta\left(Q_{0}, \theta\right),
$$

and there exists exactly one $c_{s}$ such that $c_{s}=\max \left\{c_{j}, j=0,1, \ldots, k-1\right\}$. Let $c_{0}=1$, $\delta_{1}=\max \left\{\delta\left(P_{0}, \theta\right), \delta\left(Q_{0}, \theta\right)\right\}$. We split our proof into two cases:

Case 1. Assume that $\delta_{1}>0$. By Lemma 2.3, for any given

$$
0<\varepsilon<\min \left\{n-\rho_{1}, \frac{1}{2}\left(\frac{c_{s}-c_{j}}{c_{s}+c_{j}}\right), j \neq s\right\},
$$

there exists a set $E_{2} \subset[1,+\infty)$ of a finite logarithmic measure such that for all $z$ satisfying $|z|=r \notin[0,1] \cup E_{2}, r \rightarrow+\infty$ and $\arg z=\theta \in\left[\theta_{r}-\delta_{r}, \theta_{r}+\delta_{r}\right] \backslash H_{3}$, where

$$
H_{3}=\left\{\theta \in[0,2 \pi): \delta\left(P_{0}, \theta\right)=\delta\left(Q_{0}, \theta\right)\right\}
$$

is a finite set, we have

$$
\begin{aligned}
\left|A_{s, 1}(z) e^{P_{s}(z)}+A_{s, 2}(z) e^{Q_{s}(z)}\right| & \geqslant\left|A_{s, 1}(z) e^{P_{s}(z)}\right|-\left|A_{s, 2}(z) e^{Q_{s}(z)}\right| \\
& \geqslant \exp \left\{(1-\varepsilon) c_{s} \delta\left(P_{0}, \theta\right) r^{n}\right\}-\exp \left\{(1-\varepsilon) c_{s} \delta\left(Q_{0}, \theta\right) r^{n}\right\} \\
& \geqslant \frac{1}{2} \exp \left\{(1-\varepsilon) c_{s} \delta_{1} r^{n}\right\}, \\
\left|A_{j, 1}(z) e^{P_{j}(z)}+A_{j, 2}(z) e^{Q_{j}(z)}\right| & \leqslant\left|A_{j, 1}(z) e^{P_{j}(z)}\right|+\left|A_{j, 2}(z) e^{Q_{j}(z)}\right| \\
& \leqslant \exp \left\{(1+\varepsilon) c_{j} \delta\left(P_{0}, \theta\right) r^{n}\right\}+\exp \left\{(1+\varepsilon) c_{j} \delta\left(Q_{0}, \theta\right) r^{n}\right\} \\
& \leqslant 2 \exp \left\{(1+\varepsilon) c_{j} \delta_{1} r^{n}\right\}, j=0,1, \ldots, k-1, j \neq s .
\end{aligned}
$$

By (1.5) we have

$$
\begin{aligned}
& \left|A_{s, 1}(z) e^{P_{s}(z)}+A_{s, 2}(z) e^{Q_{s}(z)}\right| \\
& \leqslant\left|\frac{f}{f^{(s)}}\right|\left\{\left|\frac{F(z)}{f}\right|+\left|\frac{f^{(k)}}{f}\right|+\sum_{j=0, j \neq s}^{k-1}\left\{\left|A_{j, 1}(z) e^{P_{j}(z)}+A_{j, 2}(z) e^{Q_{j}(z)}\right|\left|\frac{f^{(j)}}{f}\right|\right\}\right\} .
\end{aligned}
$$

Since $f$ is transcendental, from Lemma 2.2 , there exists a set $E_{1} \subset(1,+\infty)$ with $m_{l}\left(E_{1}\right)<+\infty$ and constant $B>0$, such that for all $z$ satisfying $|z|=r \notin E_{1}$, we have (3.5) holds and by Lemma 2.11, there exists a set $E_{8}$ of finite logarithmic measure such that $|z|=r \notin E_{8}$, 
$|g(z)|=M(r, g)$ and for $r$ sufficiently large inequality (3.6) holds. We know that $f$ is transcendental with $\rho(f) \geqslant n$, and by the assumptions, the poles of $f$ are of uniformly bounded multiplicities. By Hadamard factorization theorem, we can express $f$ as $f(z)=\frac{g(z)}{d(z)}$, where $g(z)$ and $d(z)$ are entire functions with

$$
\lambda(d)=\rho(d)=\lambda\left(\frac{1}{f}\right)<n, \quad \rho(g)=\rho(f) \geqslant n .
$$

Let $\rho_{1}=\max \{\rho(F), \rho(d)\}<n$. Since $|g(z)|=M(r, g) \geqslant 1$, then, by Lemma 2.4 we obtain

$$
\left|\frac{F(z)}{f(z)}\right|=\left|\frac{d(z) F(z)}{g(z)}\right|=\frac{|d(z) F(z)|}{M(r, g)} \leqslant \exp \left(r^{\rho_{1}+\varepsilon}\right) \exp \left(r^{\rho_{1}+\varepsilon}\right)=\exp \left(2 r^{\rho_{1}+\varepsilon}\right)
$$

as $|z|=r \notin[0,1] \cup E_{3}, r \rightarrow+\infty$.

By (3.5), (3.6), 4.14), 4.15), 4.16) and (4.17), for all $z$ satisfying $|z|=r_{m} \notin$ $\notin[0,1] \cup E_{1} \cup E_{3} \cup E_{8}, r_{m} \rightarrow+\infty,|g(z)|=M\left(r_{m}, g\right)$ and $\arg z=\theta \in\left[\theta_{r}-\delta_{r}, \theta_{r}+\delta_{r}\right] \backslash H_{3}$, we have

$$
\begin{aligned}
\frac{1}{2} \exp \left\{(1-\varepsilon) c_{s} \delta_{1} r_{m}^{n}\right\} \leqslant r_{m}^{2 s}\{ & \exp \left(2 r_{m}^{\rho_{1}+\varepsilon}\right)+B\left[T\left(2 r_{m}, f\right)\right]^{k+1} \\
& \left.+B\left[T\left(2 r_{m}, f\right)\right]^{k+1} \sum_{j=0, j \neq s}^{k-1} 2 \exp \left\{(1+\varepsilon) c_{j} \delta_{1} r_{m}^{n}\right\}\right\} \\
\leqslant & 4 r_{m}^{2 s} \exp \left(2 r_{m}^{\rho_{1}+\varepsilon}\right) B\left[T\left(2 r_{m}, f\right)\right]^{k+1} \sum_{j=0, j \neq s}^{k-1} \exp \left\{(1+\varepsilon) c_{j} \delta_{1} r_{m}^{n}\right\}
\end{aligned}
$$

which gives

$$
\exp \left\{(1-\varepsilon) c_{s} \delta_{1} r_{m}^{n}\right\} \leqslant 8 r_{m}^{2 s} \exp \left(2 r_{m}^{\rho_{1}+\varepsilon}\right) B\left[T\left(2 r_{m}, f\right)\right]^{k+1} \sum_{j=0, j \neq s}^{k-1} \exp \left\{(1+\varepsilon) c_{j} \delta_{1} r_{m}^{n}\right\}
$$

Since $\varepsilon<\min \left\{n-\rho_{1}, \frac{1}{2}\left(\frac{c_{s}-c_{j}}{c_{s}+c_{j}}\right), j \neq s\right\}$ is arbitrary, so by Lemma 2.6 and 4.18 we obtain

$$
\rho(f)=\limsup _{r_{m} \rightarrow+\infty} \frac{\log ^{+} T\left(r_{m}, f\right)}{\log r_{m}}=+\infty
$$

and

$$
\rho_{2}(f)=\limsup _{r_{m} \rightarrow+\infty} \frac{\log \log T\left(r_{m}, f\right)}{\log r_{m}} \geqslant n .
$$

In addition, by Lemma 2.7 and equation 1.5 , we have $\rho_{2}(f) \leqslant n$, so $\rho_{2}(f)=n$. Then, each meromorphic solution to (1.5) with poles of uniformly bounded multiplicities is of infinite order and satisfies $\rho_{2}(f)=n$.

Case 2. Assume that $\delta_{1}<0$. By Lemma 2.3, for any given $\varepsilon>0$ we obtain

$$
\begin{aligned}
\left|A_{j, 1}(z) e^{P_{j}(z)}+A_{j, 2}(z) e^{Q_{j}(z)}\right| & \leqslant\left|A_{j, 1}(z) e^{P_{j}(z)}\right|+\left|A_{j, 2}(z) e^{Q_{j}(z)}\right| \\
& \leqslant \exp \left\{(1-\varepsilon) c_{j} \delta\left(P_{0}, \theta\right) r^{n}\right\}+\exp \left\{(1-\varepsilon) c_{j} \delta\left(Q_{0}, \theta\right) r^{n}\right\} \\
& \leqslant 2 \exp \left\{(1-\varepsilon) c_{j} \delta_{1} r^{n}\right\}, j=0,1,2, \ldots, k-1 .
\end{aligned}
$$

By 1.5 we get

$$
1 \leqslant\left|\frac{f}{f^{(k)}}\right|\left(\left|\frac{F(z)}{f(z)}\right|+\sum_{j=0}^{k-1}\left\{\left|A_{j, 1}(z) e^{P_{j}(z)}+A_{j, 2}(z) e^{Q_{j}(z)}\right|\left|\frac{f^{(j)}}{f}\right|\right\}\right) .
$$


As in Case 1, by (3.5), (3.6), (4.17), 4.19) and (4.20), for all $z$ satisfying $|z|=r_{m} \notin[0,1] \cup E_{1} \cup$ $E_{3} \cup E_{8}, r_{m} \rightarrow+\infty$, at which $|g(z)|=M\left(r_{m}, g\right)$, and $\arg z=\theta \in\left[\theta_{r}-\delta_{r}, \theta_{r}+\delta_{r}\right] \backslash H_{3}$, we have

$$
1 \leqslant r_{m}^{2 k}\left(\exp \left(2 r_{m}^{\rho_{1}+\varepsilon}\right)+\sum_{j=0}^{k-1} B\left[T\left(2 r_{m}, f\right)\right]^{k+1} 2 \exp \left\{(1-\varepsilon) c_{j} \delta_{1} r_{m}^{n}\right\}\right) .
$$

Since $c_{j} \geqslant 1, j=0, \ldots, k-1, r_{m}>R_{1}>1$ and $\delta_{1}<0$, we obtain

$$
\exp \left\{(1-\varepsilon) c_{j} \delta_{1} r_{m}^{n}\right\} \leqslant \exp \left\{(1-\varepsilon) \delta_{1} r_{m}^{n}\right\}, j=0, \ldots, k-1
$$

so 4.21) becomes

$$
1 \leqslant 2 r_{m}^{2 k}(k+1) \exp \left(r_{m}^{\rho_{1}+\varepsilon}\right) B\left[T\left(2 r_{m}, f\right)\right]^{k+1} \exp \left\{(1-\varepsilon) \delta_{1} r_{m}^{n}\right\}
$$

which gives

$$
\exp \left\{(\varepsilon-1) \delta_{1} r_{m}^{n}-r_{m}^{\rho_{1}+\varepsilon}\right\} \leqslant 2 r_{m}^{2 k}(k+1) B\left[T\left(2 r_{m}, f\right)\right]^{k+1} .
$$

By Lemma 2.6 and 4.22 we obtain

$$
\rho(f)=\limsup _{r_{m} \rightarrow+\infty} \frac{\log T\left(r_{m}, f\right)}{\log r_{m}}=+\infty
$$

and

$$
\rho_{2}(f)=\limsup _{r_{m} \rightarrow+\infty} \frac{\log \log T\left(r_{m}, f\right)}{\log r_{m}} \geqslant n .
$$

In addition, by Lemma 2.7 and equation 1.5 we get $\rho_{2}(f) \leqslant n$ and hence, $\rho_{2}(f)=n$. Then, each meromorphic solution to (1.5) with poles of uniformly bounded multiplicities is of infinite order and satisfies $\rho_{2}(f)=n$.

\section{ACKNOWLEDGEMENTS}

The authors are grateful to the referee for his/her careful reading of this paper.

\section{BIBLIOGRAPHY}

1. M. Andasmas and B. Belaïdi. On the order and hyper-order of meromorphic solutions of higher order linear differential equations // Hokkaido Math. J. 42:3, 357-383 (2013).

2. B. Belaïdi and H. Habib. Relations between meromorphic solutions and their derivatives of differential equations and small functions // Ann. Univ. Buchar. Math. Ser. (LXIV) 6:1, 35-57 (2015).

3. Z. X. Chen. On the hyper-order of solutions of some second order linear differential equations // Acta Math. Sin. (Engl. Ser.) 18:1, 79-88 (2002).

4. Z. X. Chen. On the rate of growth of meromorphic solutions of higher order linear differential equations // Acta Math. Sinica (Chin. Ser.) 42:3, 551-558 (1999).

5. Z. X. Chen. The zero, pole and order of meromorphic solutions of differential equations with meromorphic coefficients // Kodai Math. J. 19:3, 341-354 (1996).

6. Z. X. Chen. Zeros of meromorphic solutions of higher order linear differential equations // Analysis 14:4, 425-438 (1994).

7. J. Chen and J. F. Xu. Growth of meromorphic solutions of higher order linear differential equations // Electron. J. Qual. Theory Differ. Equ. 2009:1, 1-13 (2009).

8. G. G. Gundersen. Estimates for the logarithmic derivative of a meromorphic function, plus similar estimates // J. London Math. Soc. 37: 1, 88-104 (1988).

9. G. G. Gundersen. Finite order solutions of second order linear differential equations // Trans. Amer. Math. Soc. 305:1, 415-429 (1988).

10. H. Habib and B. Belaïdi. On the growth of solutions of some higher order linear differential equations with entire coefficients // Electron. J. Qual. Theory Differ. Equ. 2011:93, 1-13 (2011). 
11. K. Hamani and B. Belaïdi. On the hyper-order of solutions of a class of higher order linear differential equations // Bull. Belg. Math. Soc. Simon Stevin 20:1, 27-39 (2013).

12. W. K. Hayman. Meromorphic functions // Oxford Mathematical Monographs Clarendon Press, Oxford (1964).

13. J. Jank and L. Volkmann. Einführung in die Theorie der ganzen und meromorphen Funktionen mit Anwendungen auf Differentialgleichungen // Birkhäuser Verlag, Basel, (1985).

14. K. H. Kwon. On the growth of entire functions satisfying second order linear differential equations // Bull. Korean Math. Soc 33:3, 487-496 (1996).

15. I. Laine. Nevanlinna theory and complex differential equations // de Gruyter Studies in Mathematics, 15. Walter de Gruyter \& Co., Berlin, (1993).

16. J. Tu, H. Y. Xu, H. M. Liu and Y. Liu. Complex oscillation of higher order Linear differential equations with coefficients being lacunary series of finite iterated order // Abstr. Appl. Anal. 2013, id 634739 (2013).

17. J. Wang and H. X. Yi. Fixed points and hyper-order of differential polynomials generated by solutions of differential equations // Complex Var. Theory Appl. 48:1, 83-94 (2003).

18. C. C. Yang and H. X. Yi. Uniqueness theory of meromorphic functions // Mathematics and its Applications. 557. Kluwer Academic Publ. Group, Dordrecht (2003).

19. Y. Zhan and L. Xiao. The growth of solutions of higher order differential equations with coefficients having the same order // J. Math. Res. Appl. 35:4, 387-399 (2015).

Mansouria Saidani,

Department of Mathematics,

Laboratory of Pure and Applied Mathematics,

University of Mostaganem (UMAB),

B. P. 227 Mostaganem-(Algeria).

E-mail: saidaniman@yahoo.fr

Benharrat Belaïdi,

Department of Mathematics,

Laboratory of Pure and Applied Mathematics,

University of Mostaganem (UMAB),

B. P. 227 Mostaganem-(Algeria).

E-mail: benharrat.belaidi@univ-mosta.dz 